Article

\title{
Scattering of Surface Waves by a Three-Dimensional Cavity of Arbitrary Shape: Analytical and Experimental Studies
}

\author{
Jaesun Lee ${ }^{1} \oplus$, VanTrung Ngo ${ }^{2}$, Haidang Phan ${ }^{2, *}$, TruongGiang Nguyen ${ }^{3}$, Duy Kien Dao ${ }^{4}$ and \\ Younho Cho ${ }^{5, *}$ \\ 1 School of Mechanical Engineering, Changwon National University, Changwon 51140, Korea; \\ jaesun@changwon.ac.kr \\ 2 Institute of Theoretical and Applied Research, Duy Tan University, Hanoi 100000, Vietnam; \\ trungngo.maths@gmail.com \\ 3 Institute of Mechanics, Vietnam Academy of Science and Technology, Hanoi 100000, Vietnam; \\ ntgiang@imech.vast.vn \\ 4 Faculty of Civil Engineering, Ho Chi Minh City University of Technology and Education, \\ Ho Chi Minh City 700000, Vietnam; kiendd@hcmute.edu.vn \\ 5 School of Mechanical Engineering, Pusan National University, Busan 46241, Korea \\ * Correspondence: haidangphan.vn@gmail.com (H.P.); mechcyh@pusan.ac.kr (Y.C.); \\ Tel.: +84-94-490-7624 (H.P.); +82-51-510-3206 (Y.C.)
}

Received: 20 October 2019; Accepted: 8 December 2019; Published: 12 December 2019 updates

\begin{abstract}
The scattering of surface waves by a three-dimensional shallow cavity of arbitrary shape at the surface of a homogenous, isotropic, linearly elastic half-space is theoretically investigated. A novel analytical approach based on a reciprocity consideration is introduced in this article to determine the particle displacements of the scattered wave field generated by the interaction between the surface waves and the cavity. In the usual manner, the scattered field was shown to be equivalent to the radiation from the distribution of tractions, calculated from the incident wave, on the surface of the cavity. The radiation of surface waves subjected to the computed tractions applied at a single location was found using reciprocity theorems. The field scattered by the cavity was subsequently obtained from the superposition of displacements due to all the forces applied on the cavity surface. Solutions for the scattering of surface waves by a spherical, a circular cylindrical (coin-shaped) and a square cylindrical cavity are presented in detail. We here derive the closed-form expressions of the displacement amplitudes, which represent the far-field scattered waves produced by each of the cavities. An experimental setup using the ultrasonic pulse-echo technique was then carried out to record the scattered echoes of surface waves from these cavities in order to provide practical validation of the analytical findings. The vertical displacements measured at a significant distance of about twenty-five wavelengths from the cavities of the same width and different depth were compared with the corresponding theoretical predictions. The comparisons show excellent agreement for the case of a spherical cavity and good agreement in the cases of a circular and a cylindrical cavity in terms of trends and magnitudes. It is followed by a discussion on the results of the comparison and the limitations of the proposed approach regarding the degree of smoothness and the size of cavity.
\end{abstract}

Keywords: surface wave; half-space; three-dimensional cavity; reciprocity

\section{Introduction}

Understanding the interaction of elastic waves with surface defects like cracks, corrosion pits, topographic irregularities, etc., is critical for the potential development of ultrasonic nondestructive 
evaluation (NDE). However, the wave scattering problem remains a challenging area of research due to the complexity of the scattering phenomenon. For this reason, most of the works in the literature are performed with the use of numerical methods, such as the finite element (FE) method [1], the boundary element (BE) method [2], or the finite element boundary integral (FE-BI) approach [3]. For a three-dimensional (3D) scattering problem, investigation with numerical methods is highly computationally expensive. Computational times remain of the order of several hours for one particular incident wavefield, even on powerful machines. Analytical or semi-analytical solutions to such problems are therefore desirable. They provide independent validation of numerical methods and enable rapid simulations of scattering from large populations of different scatterers. They also enable the relative contributions of different modes (e.g., propagating or non-propagating) to be examined to provide insight into the physics of the scattering process.

For the present paper, we studied the general three-dimensional problem of surface wave scattering by a cavity of arbitrary shape on the surface of a half-space. Two approximate methods using concentrated loading and distributed loading for the theoretical analysis of the scattered field are introduced. Examples of calculations include the scattering of surface waves by a spherical, a circular cylindrical (coin-shaped), and a square cylindrical cavity. Experimental work was carried out to verify the analytical solutions and it is followed by a discussion on the results of comparison. The investigation is the very first theoretical approach for obtaining the solutions of the scattering of surface waves by a three-dimensional cavity of arbitrary shape. Closed-form expressions that represent the scattered wave field due to the interaction with the cavity are derived. For these explicit solutions, computation of the scattered amplitudes is extremely simple. The approximation models can offer a much lower computational cost and time with a reasonable accuracy in comparison with the existing numerical methods. They are also able to provide insight into the physics of the scattering process that is important to solving inverse scattering problems.

With respect to earlier work, much of the work in the literature has been concerned with scattering by two-dimensional surface-breaking cracks. Typical examples of analytical work are References [4-6]. Semi-analytical solutions were obtained, for example, in References $[7,8]$. Numerical work was carried out using the finite element method [1] and the boundary element method (see References $[9,10]$ ). The scattering of surface waves by a two-dimensional cavity was theoretically investigated in our earlier works [11-13]. Defect detection in rails using surface waves was also studied by Hesse and Cawley [14]. In a related category are papers on scattering by strips and grooves [15-19]. A review of the scattering of elastic waves by defects was presented in Martin [20] (pp. 141-143). The related problem of the scattering of Lamb waves by a surface defect in a layer has received considerable attention (see, for example, References [2,21]).

In three dimensions, the scattering of elastic waves by canyons of arbitrary shape was studied using an indirect boundary integral equation method [22]. The 3D scattering of guided waves by a through-thickness cavity with an irregular shape in an isotropic plate is investigated in Moreau et al. [23] and scattering signal analysis of a cylindrical structure from a torsional wave is presented by Lee [24]. An analytical solution for the scattering of ultrasonic guided waves by flat-bottomed cavities with arbitrary shape in a plate was introduced in Moreau et al. [25]. Grahn [26] discussed the scattering of Lamb waves from a circular partly through-thickness hole.

This paper proceeds through seven sections. Section 2 states the problem as the superposition of the incident wave and the scattered field. It is shown that the scattered field is equivalent to the field radiated by surface tractions on the surface of the cavity. These surface tractions were obtained from the incident wave. Free Rayleigh surface waves propagating in a half-space are presented in Section 3. A summary of the reciprocity approach to surface wave motion used in this paper is discussed in Section 4. The analytical approach that uses distributed loading for a scattering of surface waves by a three-dimensional cavity is introduced in Section 5. Section 6 is devoted to the derivation of the scattered field by a spherical, a cylindrical (coin-shaped) and a square-shaped cavity. 
Experimental work is conducted, and the experimental measurements are compared with the analytical findings in Section 7. Major conclusions drawn from this investigation are given in Section 8.

\section{Statement of the Problem}

Consider a homogeneous, isotropic, linearly elastic half-space $z \geq 0$ in the Cartesian coordinate system $(x, y, z)$. The half-space contains a cavity on the surface as shown in Figure 1a. A two-dimensional plane surface wave propagating in the $x$-direction is incident on the cavity. The interaction of the surface wave with the cavity generates a scattered wavefield $\boldsymbol{u}_{s c}$ (Figure 1c), which is of interest and will be studied theoretically in this paper. Using the decomposition principle, which is demonstrated in Figure 1, the total wavefield $\boldsymbol{u}_{t o}$ (Figure 1a) is equal to the summation of the incident wavefield $\boldsymbol{u}_{\text {in }}$ (Figure $1 \mathrm{~b}$ ) and the scattered field $\boldsymbol{u}_{s c}$. It should be noted that the incident field considered here is a two-dimensional (2D) surface wave, while the scattered field is a 3D surface wave. The far-field displacements of 3D surface waves radiated from a cavity were determined analytically and experimentally and are presented in the next sections.
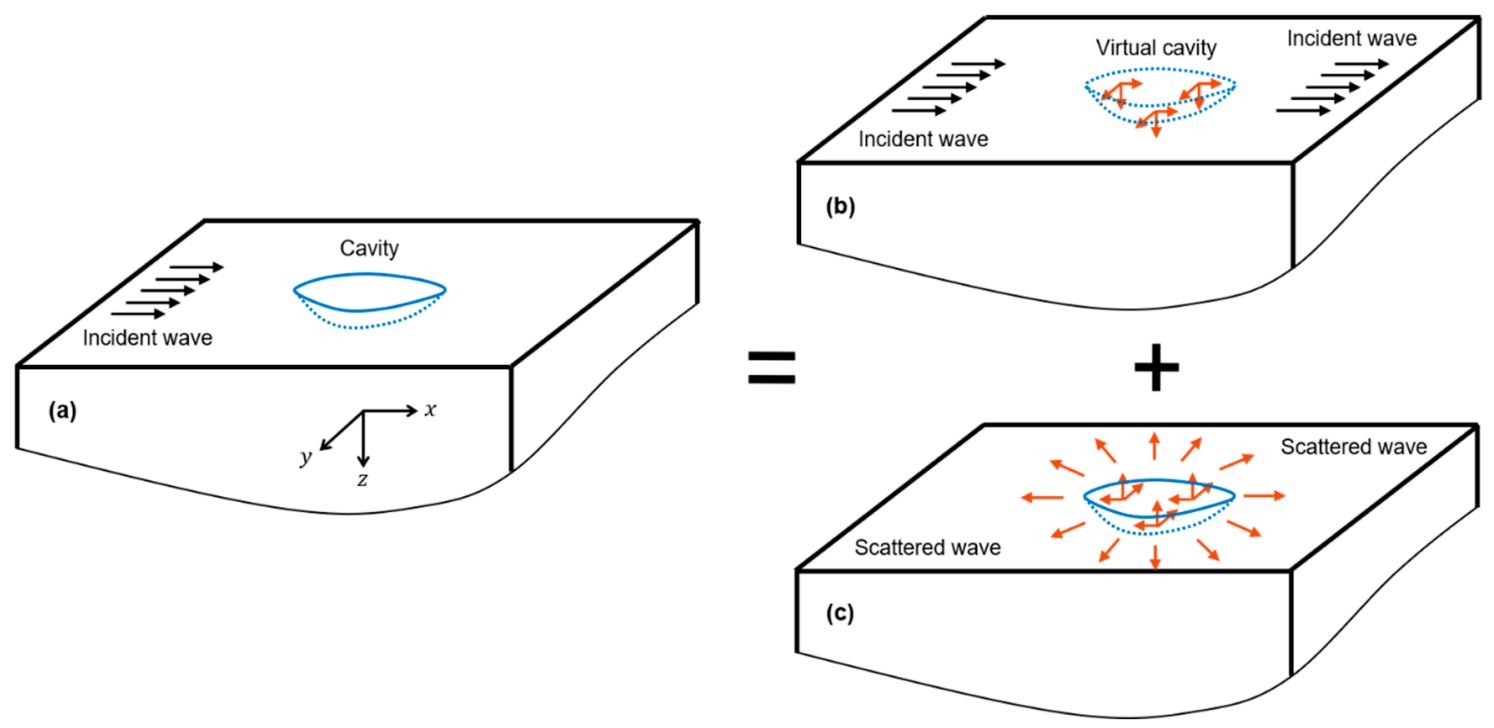

Figure 1. Linear decomposition technique: (a) the interaction of the incident surface wave with the three-dimensional cavity, (b) the propagation of the incident wave in the half-space without the cavity, and (c) the scattered field produced by the incident wave interaction with the cavity.

With the linear decomposition given in Figure 1, the scattered field is equivalent to the field generated by the application of a distribution of the tractions applied on the surface of the cavity. These traction components are equal in magnitude but opposite in sign to the corresponding tractions due to the incident wave on a virtual cavity in the half-space without the cavity. Therefore, they can be computed from the stress components of the incident Rayleigh wave and the outward normal vectors of the cavity. It should be noted that for the scattered problem given in Figure 1c, the calculated tractions will generate both body waves and three-dimensional surface waves. It was discussed in the literature (see page 55 of Ewing and Zardetzky [27] and page 321 of Achenbach [28] for details) that for the three-dimensional case, the surface waves attenuate with a distance from the point of excitation according to $r^{-1 / 2}$ while the body waves decay according to $r^{-1}$, where $r$ is the distance from the point of application. Therefore, the surface waves dominate at a sufficiently large distance from the excitation point. The displacements of the scattered surface wave field are of interest and are determined in this article.

Two analytical approximation approaches will be presented. When the depth and the width of cavities are both much smaller than the incident wavelength, the tractions acting on the cavity surface can be replaced by three resultant loads applied at the origin of the coordinate system. 
This approximation is simple in the calculation but is supposed to be less accurate when the size of the cavity (depth and width) is comparable to the incident wavelength. As an improvement, a distributed approach is considered in which the application of the distributed tractions acting on the cavity surface is shifted to the surface of the half-space. The traction components are first computed for every point of the cavity surface. The reciprocity theorem is then applied to the equivalent loads that are applied on the surface of the half-space to obtain the displacement amplitudes of the scattered field. The total displacement amplitude of the scattered field is a superposition of the amplitudes generated by the loads at every point on the surface.

\section{Free Surface Waves in a Half-Space}

The possibility of a wave traveling along the free surface of an elastic half-space such that the displacements decay exponentially with distance from the surface was first considered by Rayleigh [29]. Additional related studies can be found, e.g., in the pages 187-194 of the textbook by Achenbach [28]. A surface wave, also called a Rayleigh wave, is defined by an angular frequency $\omega$ and a wavenumber $k=\omega / c$, where $c$ is the surface wave velocity; other values include the Lame constants $\lambda, \mu$ and the mass density $\rho$. For a Cartesian coordinate system $(x, y, z)$, the displacements of the incident surface waves along the half-space $z \geq 0$ in the positive $x$-direction are of the forms:

$$
u_{x}=i A\left(d_{1} e^{-k p z}+d_{2} e^{-k q z}\right) e^{i(k x-\omega t)}, \quad u_{z}=A\left(d_{3} e^{-k p z}-e^{-k q z}\right) e^{i(k x-\omega t)},
$$

where $A$ is the complex amplitude; $t$ indicates time; and $d_{1}, d_{2}, d_{3}, p, q$ are dimensionless quantities. For an elastic half-space with the material properties given, the well-known equation for the phase velocity of surface waves (see page 32 of Achenbach [28]) has a unique solution independent of the wavenumber. These dimensionless quantities, therefore, depend only on the material properties of the half-space. They are not presented here for the purpose of simplicity but curious readers can find their expressions in, for example, Phan et al. [30]. Note that the stress components can be easily calculated from the displacements with the help of Hooke's law.

\section{Surface Wave Motion Generated by a Time-Harmonic Point Load}

This is a three-dimensional problem. The surface wave motion generated by a time-harmonic point load can be calculated in a simple manner using the reciprocity theorem, with input being the actual surface wave with an unknown amplitude and a virtual free surface wave [30,31]. The method requires expressions for the displacements and the stresses of free surface waves, preferably in an analytical form, but numerically obtained forms can also be used. Suppose that the half-space is subjected to a time-harmonic point load at the surface pointing in an arbitrary direction. Without loss of generality, the coordinate system can be chosen such that the load acts in the $x z$-plane at the origin of the system. The surface wave response is then sought as the superposition of the responses due to the normal component of magnitude $P$ in the $z$-direction and the horizontal component of magnitude $Q$ in the $x$-direction.

For surface wave motions radiated from a point load, it is convenient to also use a cylindrical coordinate $(r, \theta, z)$ defined by $x=r \cos \theta, y=r \sin \theta$, and $z=z$ along with the Cartesian coordinates $(x, y, z)$. Solutions of surface wave motion by the reciprocity approach are obtained in Phan et al. [30]. Here, we summarize the results, which are later used for solving the three-dimensional scattering problem. The vertical displacement of surface waves generated by a vertical loading $P$ in the $z$-direction may be written as:

$$
u_{z}^{P}=\frac{k}{4 i} \frac{P W(0)}{\mu E} W(z) H_{0}^{(1)}(k r)
$$


For a horizontal load $Q$ in the $x$-direction, the generated displacement is of the form:

$$
u_{z}^{Q}=\frac{k}{4 i} \frac{Q U(0)}{\mu E} W(z) H_{1}^{(1)}(k r) \cos \theta .
$$

In Equations (2) and (3), $H_{j}^{(i)}(\xi)$ represents the $j^{\text {th }}$ order Hankel function of the $i^{\text {th }}$ kind and $E$ is a dimensionless quantity determined using:

$$
E=\left(\frac{d_{4} d_{1}-d_{6} d_{3}}{2 p}+\frac{d_{4} d_{2}+d_{5} d_{1}-d_{7} d_{3}+d_{6}}{p+q}+\frac{d_{2} d_{5}+d_{7}}{2 q}\right)
$$

\section{Scattering of Surface Waves by a Cavity of Arbitrary Shape}

A plane surface wave propagating in the $x$-direction is incident on a three-dimensional cavity of arbitrary shape at the surface of an isotropic homogeneous elastic half-space. The incidence generates a scattered field that is of interest in this article. This section introduces two approximations to the theoretical analysis of the scattered field. Consider a cavity on the surface of a half-space as given in Figure 2. In the coordinate system $(x, y, z)$, suppose that the surface of the cavity $S$ is defined by $z=h(x, y)$. Then the outward normal vector $n\left(n_{x}, n_{y}, n_{z}\right)$ of $d S$ can be found using:

$$
\boldsymbol{n}=\frac{\left(h_{x}^{\prime}, h_{y^{\prime}}^{\prime}-1\right)}{\sqrt{\left(h_{x}^{\prime}\right)^{2}+\left(h_{y}^{\prime}\right)^{2}+1}}
$$

where $h_{x}^{\prime}=\frac{\partial h(x, y)}{\partial x}$ and $h_{y}^{\prime}=\frac{\partial h(x, y)}{\partial y}$.

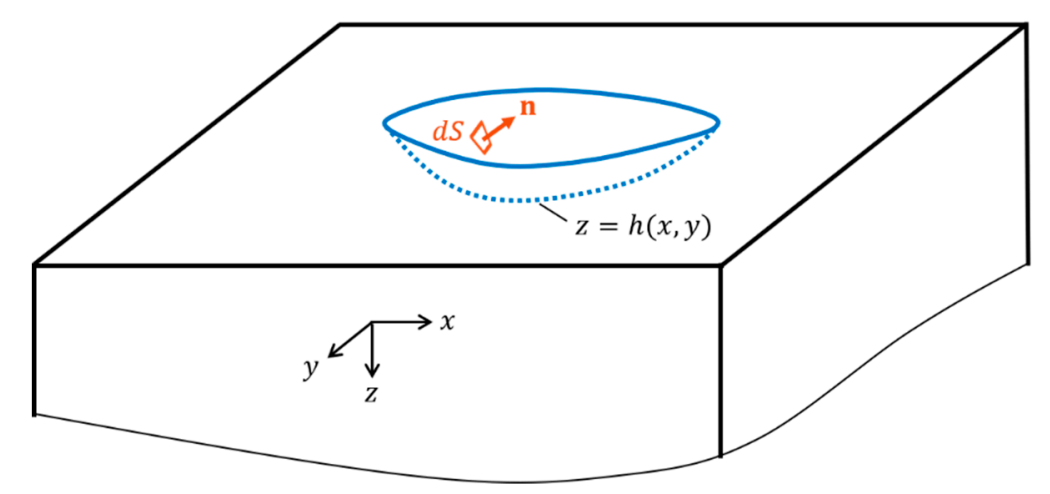

Figure 2. A cavity in a half-space.

From the linear decomposition principle, as stated in Section 2, the scattered field is equivalent to the field generated by the application of the distribution of tractions on the surface of the cavity, as shown in Figure 1c. These tractions are equal in magnitude but opposite in sign to the corresponding tractions due to the incident wave on a virtual cavity in the half-space without the cavity, as shown in Figure $1 \mathrm{~b}$. Thus, they are equal to $-t$ where $t_{i}(i=x, y, z)$ are determined from the stress components of the incident Rayleigh wave and the outward normal vector as $t_{i}=\tau_{j i} n_{j}$. The tractions, in turn, generate the scattered field, which is of concern in this paper. It should be noted that these tractions generate body waves, as well as surface waves radiating from the cavity. In the three-dimensional scattering, the surface waves attenuate with the distance from the points of excitation on the cavity according to $r^{-1 / 2}$ while the body waves decay according to $r^{-1}$, where $r$ is the distance from the cavity. Therefore, the surface waves dominate at a sufficiently large distance from the cavity. 


\subsection{Concentrated Loading Approximation}

As a simple approximation, the tractions on the surface of the cavity are replaced by three equivalent resultant forces applied at the origin of the coordinate system in the same directions. These forces then generate a scattered field of surface waves in the half-space. This approximation is supposed to be valid only when the depth and the width of the cavity are very small in comparison with the wavelength.

For an incident surface wave propagating in the positive $x$-direction with amplitude $A_{\text {in }}$, the resultant forces $F\left(F_{x}, F_{y}, F_{z}\right)$ are obtained via integration of the tractions over the surface $S$. These forces generate a field that approximates the scattered field. The total vertical displacement of the scattered field in the $z$-direction is the summation of the displacements generated by the resultant forces $F_{x}, F_{y}, F_{z}$ and is written as:

$$
u_{z}=\frac{k W(z)}{4 i \mu E}\left[U(0)\left(F_{x} \cos \theta+F_{y} \sin \theta\right) H_{1}^{(1)}(k r)+W(0) F_{z} H_{0}^{(1)}(k r)\right] .
$$

\subsection{Distributed Loading Approximation}

An approximation using distributed loading is now considered. The tractions first calculated at every point on the surface of the cavity are shifted to the surface of the half-space. The reciprocity theorem is then applied for these equivalent surface loads to obtain the displacement amplitudes of the scattered field. In the concentrated loading approximation, the loads that generate the scattered field are all applied at the origin of the coordinate system. For the current approximation, the loads are applied over an area, which is the projection of the cavity on the surface of the half-space. This method is believed to be valid for shallow cavities. The obtained theoretical predictions were compared with the experimental results in order to understand the limitations of the proposed approach.

Suppose that a point source is applied at $\left(x_{0}, y_{0}\right)$, or $\left(r_{0}, \theta_{0}\right)$ using cylindrical coordinates, and the observation point is located at $(r, 0)$, as illustrated in Figure 3 . Here, the observation point is chosen with $\theta=0$ for the purpose of simplicity. Note that solutions for other points, i.e., $\theta \neq 0$ can be easily gained by using Equations (2) and (3). The following relations are found in Figure 3 for the case of $\theta=0$ :

$$
r^{\prime}=\sqrt{r_{0}^{2}+r^{2}-2 r r_{0} \cos \theta_{0}}, \quad \cos \theta \prime=\frac{r-r_{0} \cos \theta_{0}}{\sqrt{r_{0}^{2}+r^{2}-2 r r_{0} \cos \theta_{0}}} .
$$

For an incident surface wave propagating in the positive $x$-direction with amplitude $A_{\text {in }}$, the point forces corresponding to the tractions on the surface of the virtual cavity at $\left(x_{0}, y_{0}, z_{0}\right)$ can be calculated using:

$$
f_{i}\left(x_{0}, y_{0}, z_{0}\right)=-t_{i}\left(x_{0}, y_{0}, z_{0}\right) d S, \quad i=x, y, z .
$$

It is convenient to also represent the expressions of $f_{i}\left(x_{0}, y_{0}, z_{0}\right)$ and $t_{i}\left(x_{0}, y_{0}, z_{0}\right)$ given by Equation (8) in the cylindrical coordinate $(r, \theta, z)$. For a time-harmonic load at the origin of the coordinate system, the vertical displacements follow from Equations (2) and (3). In the case of a time-harmonic load applied at $\left(r_{0}, \theta_{0}, 0\right)$ as in Figure 3, the total vertical displacement of the scattered field at the observation point $(r, 0, z)$ is the summation of the surface wave fields generated by $f_{i}(i=x, y, z)$. Thus:

$$
u_{z}=\frac{-k W(z)}{4 i \mu E} \iint_{S}\left[U(0)\left(t_{x} \cos \theta \prime+t_{y} \sin \theta \prime\right) H_{1}^{(1)}(k r \prime)+W(0) t_{z} H_{0}^{(1)}(k r \prime)\right] d S .
$$

It should be noted that the displacement given in Equation (9) is dependent on frequency since its expression appears with a frequency term via the wavenumber $k$. 


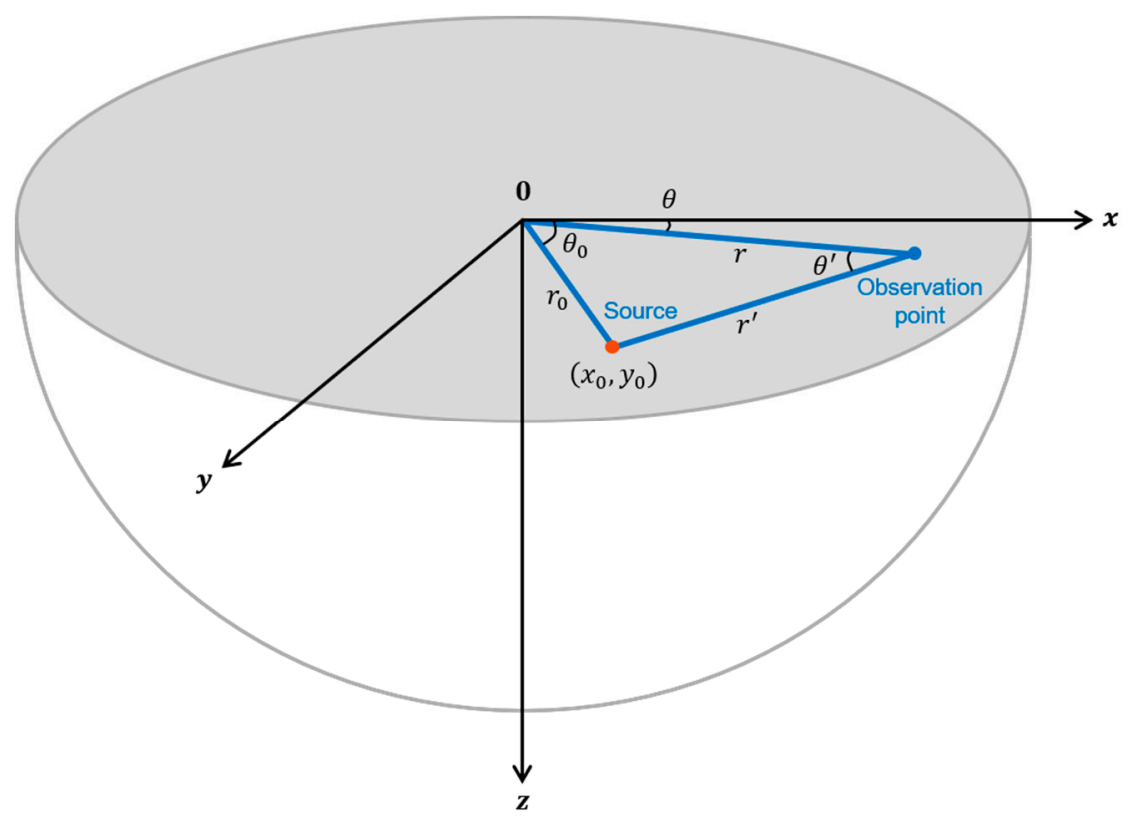

Figure 3. Source and observation point.

\section{Computation of the Scattered Fields from a Spherical, a Circular, and a Square Cylindrical Cavity}

\subsection{Spherical Cavity}

Consider a three-dimensional spherical cavity on the surface of an elastic half-space, as demonstrated in Figure 4. The cavity has a depth $D$, a width $2 R_{0}$, and a cavity radius $R$. The area $S$ of the surface trace is defined as $S=\left\{x, y \mid x^{2}+y^{2} \leq R_{0}^{2}\right\}$. In the coordinate system $(x, y, z)$, we have:

$$
R^{2}=x^{2}+y^{2}+(z+Z)^{2}, Z=R-D, R^{2}=R_{0}^{2}+Z^{2}
$$

We define the dimensionless quantities $\bar{r}_{0}=k r_{0}, \bar{R}=k R, \bar{R}_{0}=k R_{0}, \bar{D}=k D$, and $\bar{z}_{0}=k z_{0}$, which will be used for the expressions of the vertical displacement of scattered wave field.

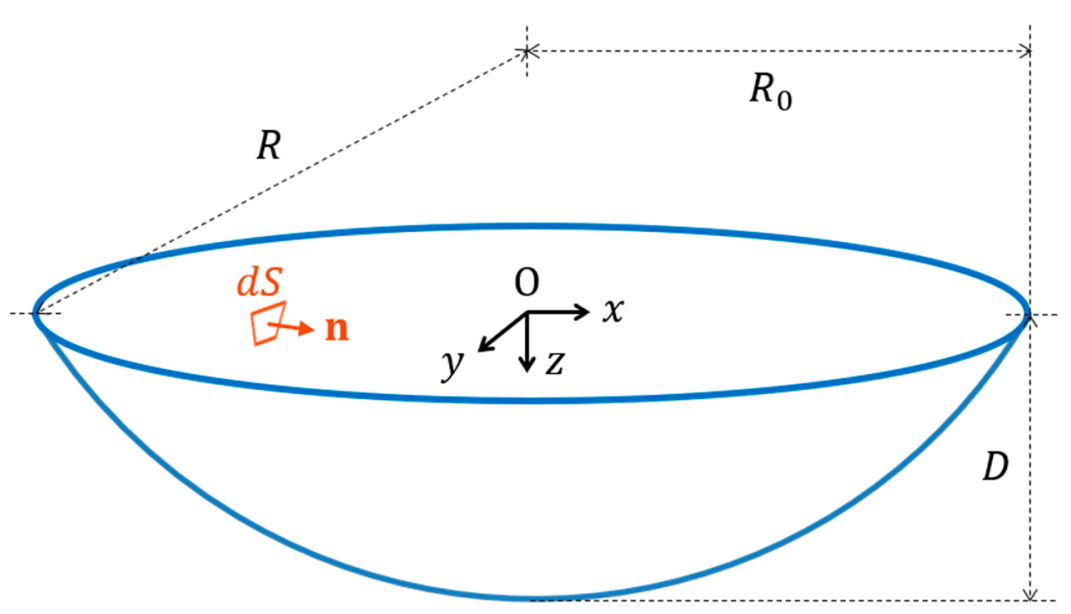

Figure 4. A spherical cavity in a half-space.

The cylindrical coordinates are used for the computation of the scattered field. The total vertical displacement for $\theta=0$ is computed using: 


$$
u_{z}=-\frac{A_{\text {in }}}{4 i t} W(z)\left\{\int_{0}^{\bar{R}_{0}} \int_{0}^{2 \pi}\left[U(0) \bar{f}_{x}\left(\bar{r}_{0}, \theta_{0}, \bar{z}_{0}\right) H_{1}^{(1)}\left(\bar{r}^{\prime}\right) \cos \theta^{\prime}+U(0) \bar{f}_{y}\left(\bar{r}_{0}, \theta_{0}, \bar{z}_{0}\right) H_{1}^{(1)}\left(\bar{r}^{\prime}\right) \sin \theta^{\prime}+W(0) \bar{f}_{z}\left(\bar{r}_{0}, \theta_{0}, \bar{z}_{0}\right) H_{0}^{(1)}\left(\bar{r}^{\prime}\right)\right] d \bar{r}_{0} d \theta_{0}\right\},
$$

where

$$
\begin{aligned}
\bar{f}_{x}\left(\bar{r}_{0}, \theta_{0}, \bar{z}_{0}\right)= & \left(\frac{\bar{r}_{0} \cos \theta_{0}}{\sqrt{\bar{R}^{2}-\bar{r}_{0}^{2}}}\left(d_{4} e^{-p \bar{z}_{0}}+d_{5} e^{-q \bar{z}_{0}}\right)+i\left(d_{6} e^{-p \bar{z}_{0}}+d_{7} e^{-q \bar{z}_{0}}\right)\right) e^{i \bar{r}_{0} \cos \theta_{0} \bar{r}_{0}} \\
& \bar{f}_{y}\left(\bar{r}_{0}, \theta_{0}, \bar{z}_{0}\right)=\left(\frac{\bar{r}_{0} \sin \theta_{0}}{\sqrt{\bar{R}^{2}-\bar{r}_{0}^{2}}} d_{10} e^{-p \bar{z}_{0}}\right) e^{i \bar{r}_{0} \cos \theta_{0} \bar{r}_{0}} \\
\bar{f}_{z}\left(\bar{r}_{0}, \theta_{0}, \bar{z}_{0}\right)= & \left(i \frac{\bar{r}_{0} \cos \theta_{0}}{\sqrt{\bar{R}^{2}-\bar{r}_{0}^{2}}}\left(d_{6} e^{-p \overline{z_{0}}}+d_{7} e^{-q \bar{z}_{0}}\right)+\left(d_{8} e^{-p \bar{z}_{0}}+d_{9} e^{-q \bar{z}_{0}}\right)\right) e^{i \bar{r}_{0} \cos \theta_{0} \bar{r}_{0}}
\end{aligned}
$$

with

$$
\begin{gathered}
\bar{r}^{\prime}=\sqrt{\bar{r}_{0}^{2}+\bar{r}^{2}-2 \overline{r r}_{0} \cos \theta_{0}} \\
\cos \theta \prime=\frac{\bar{r}-\bar{r}_{0} \cos \theta_{0}}{\sqrt{\bar{r}_{0}^{2}+\bar{r}^{2}-2 \bar{r}_{0} \cos \theta_{0}}}, \sin \theta \prime=\frac{\bar{r}_{0} \sin \theta_{0}}{\sqrt{\bar{r}_{0}^{2}+\bar{r}^{2}-2 \bar{r}_{0} \cos \theta_{0}}} .
\end{gathered}
$$

\subsection{Circular Cylindrical Cavity}

Consider now a cylindrical (coin-shaped) cavity oriented perpendicular to the surface of an elastic half-space. The cavity has a depth $D$ and the radius of the cylinder is $R_{0}$. The tractions on the bottom and lateral surfaces generate the scattered field of the surface waves. Like in the case of a spherical cavity, it is suitable to use cylindrical coordinates. In a similar procedure, the displacement is found using:

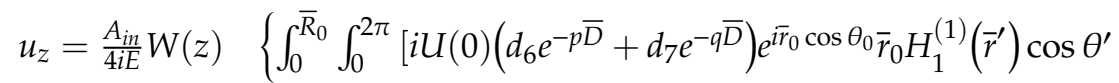

$$
\begin{aligned}
& \left.+W(0)\left(d_{8} e^{-p \bar{D}}+d_{9} e^{-q \bar{D}}\right) e^{i \bar{r}_{0} \cos \theta_{0}} \bar{r}_{0} H_{0}^{(1)}\left(\bar{r}^{\prime}\right)\right] d \bar{r}_{0} d \theta_{0} \\
& +\int_{0}^{\bar{D}} \int_{0}^{2 \pi}\left[(0)\left(d_{4} e^{-p \bar{z}_{0}}+d_{5} e^{-q \bar{z}_{0}}\right) \bar{R}_{0} \cos \theta_{0} e^{i \bar{R}_{0} \cos \theta_{0}} H_{1}^{(1)}\left(\bar{R}^{\prime}\right) \cos \Phi^{\prime}\right. \\
& +U(0) d_{10} e^{-p \overline{z_{0}}} \bar{R}_{0} e^{i \bar{R}_{0} \cos \theta_{0}} \sin \theta_{0} H_{1}^{(1)}\left(\bar{R}^{\prime}\right) \sin \Phi^{\prime} \\
& \left.\left.i W(0)\left(d_{6} e^{-p \bar{z}_{0}}+d_{7} e^{-q \bar{z}_{0}}\right) \bar{R}_{0} \cos \theta_{0} e^{i \bar{R}_{0} \cos \theta_{0}} H_{0}^{(1)}\left(\bar{R}^{\prime}\right)\right] d \bar{z}_{0} d \theta_{0}\right\},
\end{aligned}
$$

where

$$
\begin{gathered}
\bar{R}^{\prime}=\sqrt{\bar{R}_{0}^{2}+\bar{r}^{2}-2 \bar{r} \bar{R}_{0} \cos \theta_{0}}, \\
\cos \Phi \prime=\frac{\bar{r}-\bar{R}_{0} \cos \theta_{0}}{\sqrt{\bar{R}_{0}^{2}+\bar{r}^{2}-2 r \bar{R}_{0} \cos \theta_{0}}}, \sin \Phi \prime=\frac{\bar{R}_{0} \sin \theta_{0}}{\sqrt{\bar{R}_{0}^{2}+\bar{r}^{2}-2 \bar{r} \bar{R}_{0} \cos \theta_{0}}} .
\end{gathered}
$$

\subsection{Square Cylindrical Cavity}

For a square-shaped cavity on the surface of an elastic space that has a depth $D$ and a side length of square $2 R_{0}$, the tractions on the bottom and four lateral surfaces generate the scattered field of the surface waves. In this case, it is more convenient to use a Cartesian coordinate system for the integral calculation. Similarly, the vertical displacement is found using: 


$$
\begin{aligned}
u_{z}=\frac{A_{i n}}{4 i E} W(z) & \left\{\int _ { - \overline { R } _ { 0 } } ^ { \overline { R } _ { 0 } } \int _ { - \overline { R } _ { 0 } } ^ { \overline { R } _ { 0 } } \left[i U(0)\left(d_{6} e^{-p \bar{D}}+d_{7} e^{-q \bar{D}}\right) e^{i \bar{x}_{0}} H_{1}^{(1)}\left(\bar{r}^{\prime}\right) \cos \theta^{\prime}\right.\right. \\
& \left.-W(0)\left(d_{8} e^{-p \bar{D}}+d_{9} e^{-q \bar{D}}\right) e^{i \bar{x}_{0}} H_{0}^{(1)}\left(\bar{r}^{\prime}\right)\right] d \bar{x}_{0} d \bar{y}_{0} \\
& +\int_{0}^{\bar{D}} \int_{-\bar{R}_{0}}^{\bar{R}_{0}}\left[2 i U(0) \sin \bar{R}_{0}\left(d_{4} e^{-p \bar{z}_{0}}+d_{5} e^{-q \bar{z}_{0}}\right) H_{1}^{(1)}\left(\bar{r}^{\prime}\right) \cos \theta^{\prime}\right. \\
& +2 i U(0) \sin \bar{R}_{0} d_{10} e^{-p \bar{z}_{0}} H_{1}^{(1)}\left(\bar{r}^{\prime}\right) \cos \theta^{\prime} \\
& \left.\left.+2 W(0) \sin \bar{R}_{0}\left(d_{6} e^{-p \bar{z}_{0}}+d_{7} e^{-q \bar{z}_{0}}\right) H_{0}^{(1)}\left(\bar{r}^{\prime}\right)\right] d \bar{y}_{0} d \bar{z}_{0}\right\},
\end{aligned}
$$

where $\bar{x}_{0}=k x_{0}, \bar{y}_{0}=k y_{0}, \bar{z}_{0}=k z_{0}$.

\section{Experimental Confirmation and Discussion}

An experimental study was carried out in order to provide practical validation of the findings of the analytical work in the previous sections. The experimental setup using an ultrasonic pulse-echo technique is illustrated in Figure 5. The piezoelectric (PZT) transducer (Hagisonic, Daejeon, Republic of Korea) placed on the critical angle was first used to transmit a surface-wave pulse with a central frequency of $f=0.5 \mathrm{MHz}$ toward the cavity. It was then used as a receiver to record the echoes of the scattered surface waves. The distance from the transmitter to the defect was chosen as $d=150 \mathrm{~mm}$, which was about twenty-five wavelengths. At this distance, the surface waves completely dominated the scattered wave field since the body waves had almost vanished.

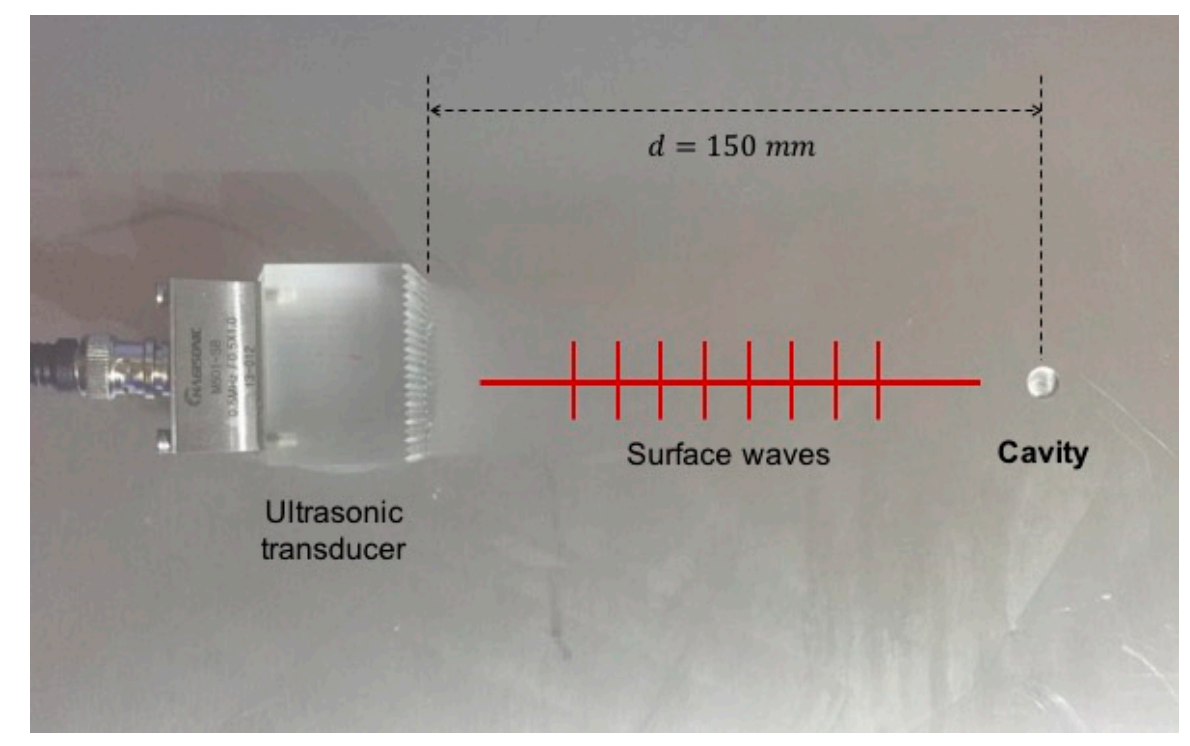

Figure 5. Experiment set-up.

A $25 \mathrm{~mm}$ thickness steel plate, which had a shear modulus of $\mu=7.9872 \times 10^{10} \mathrm{~N} / \mathrm{m}^{2}$, a Lame's constant of $\lambda=11.03 \times 10^{10} \mathrm{~N} / \mathrm{m}^{2}$, and a density of $\rho=7800 \mathrm{~kg} / \mathrm{m}^{2}$, was used for the experimental program. The thickness, which was more than four wavelengths, guaranteed that no ultrasonic signal was reflected from the bottom of the plate. The spherical, circular cylindrical, and square cylindrical cavities were made on the surface of the steel material for the experiments. Each of three shapes included ten cavities of same width and different depth. This meant that for each shape the width of cavity $2 R_{0}$ was fixed while its depth $D$ varied such that dimensionless quantity $k D$, where $k$ is the wavenumber, varied.

By recording the echoes from the defects, the displacement amplitudes of the scattered wave fields were determined. These results were then divided by the referenced amplitude of the incident surface wave, which was measured using the pitch catch technique, to obtain the normalized amplitude ratios. For each cavity configuration, this experimental process was repeated five times to calculate the means 
and the standard deviations of the normalized ratios. These experimental results were assumed to represent the scattered surface wave fields.

For the analytical study, the normalized amplitude ratio was also defined by the proportion of the displacement amplitude of the scattered field to the one of the incident field. In the following, the comparisons between the analytical results and experimental data for the absolute values of the normalized amplitude ratios are shown versus the dimensionless $k D$ for the cases of a spherical, a circular cylindrical, and a square cylindrical cavity.

The first example was the study for ten spherical cavities with $D$ varying from $D=0.3 \mathrm{~mm}$ to $D=1.2 \mathrm{~mm}$, while $R_{0}=3.0 \mathrm{~mm}$. The comparison results are exhibited in Figure 6 . The theoretical and experimental amplitude ratios were observed to increase with the increase of the cavity depth. An excellent agreement was found between the two curves. They had only about a $6-8 \%$ difference on average. The comparisons between the analytical predictions and the experimental measurements of the normalized amplitude ratios for the case of a circular cylindrical cavity and a square cylindrical cavity are shown in Figures 7 and 8, respectively. In these studies, $D$ varied between $0.3 \mathrm{~mm}$ and $1.2 \mathrm{~mm}$, while $R_{0}=4.0 \mathrm{~mm}$. A very good agreement of the trend was obtained in both sets of results. In terms of magnitude, the experimental points and the predicted points were in reasonable agreement. There were, however, some differences in the details between the two sets of calculations. It is believed that the differences were due to the analytical approximation rather than the experiment. For the case of a spherical cavity, which had a smooth surface, an excellent agreement is shown in Figure 6. As the surfaces of the circular and square cylindrical cavities were not smooth, the comparisons between the analytical and experimental results show some differences in Figures 7 and 8. It should also be realized that the analytical calculations and the experimental results increased the differences with an increase of the size (the volume) of the cavities from the spherical cavity (Figure 6) to the cylindrical cavity (Figure 7) and to the square cavity (Figure 8). This proposed approach, therefore, has limitations regarding the degree of smoothness and the size of the cavity.

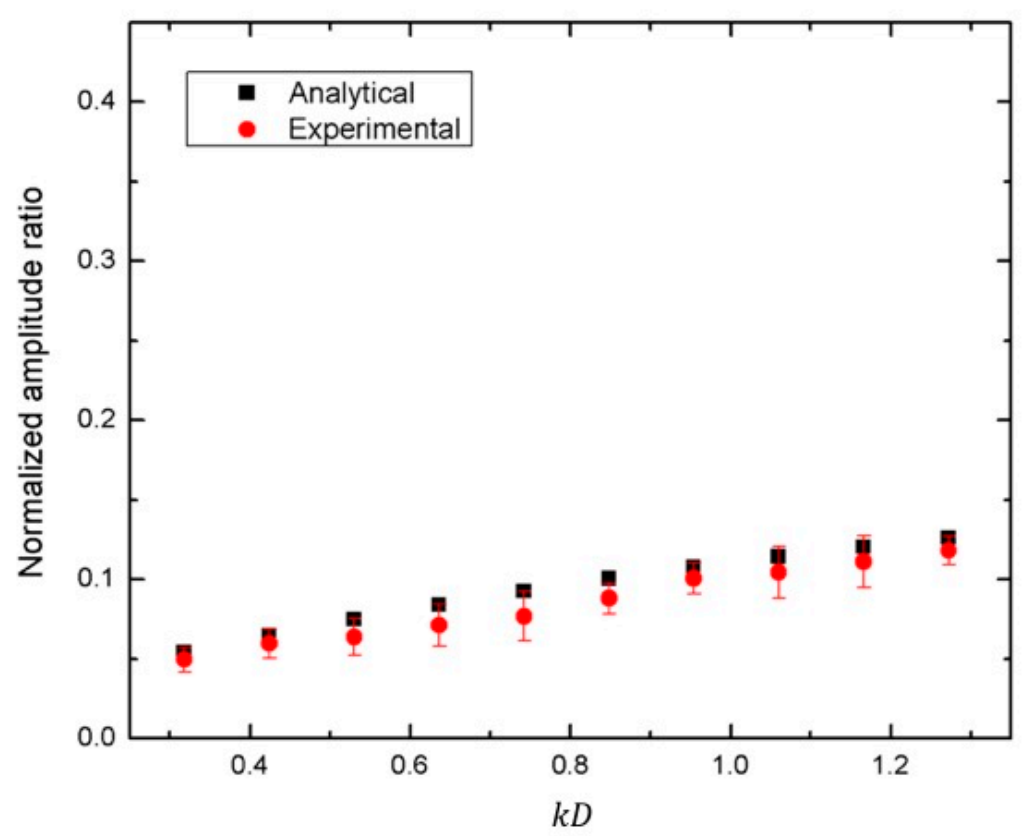

Figure 6. Normalized amplitude ratio between the scattered field and the incident field due to the interaction of the incident surface wave with a spherical cavity: $D=0.3 \mathrm{~mm}$ to $D=1.2 \mathrm{~mm}, R_{0}=3.0 \mathrm{~m}$, frequency $f=0.5 \mathrm{MHz}$. 


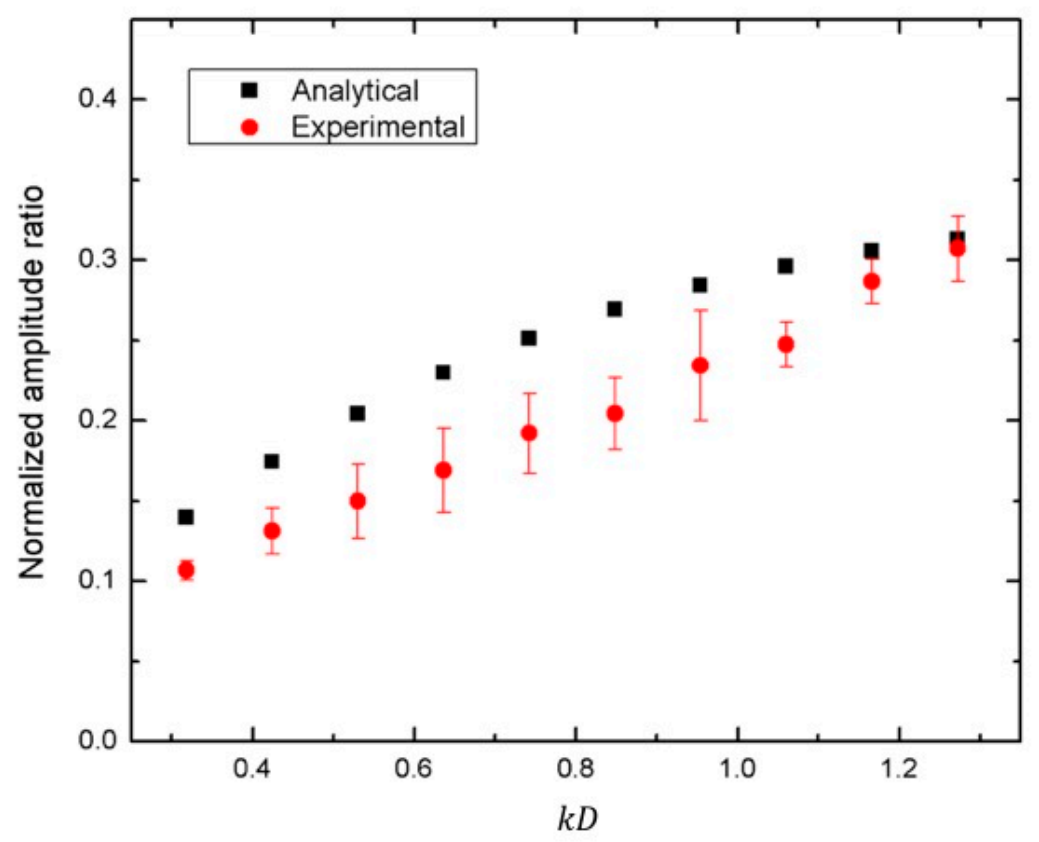

Figure 7. Normalized amplitude ratio between the scattered field and the incident field due to the interaction of the incident surface wave with a circular cylindrical cavity: $D=0.3 \mathrm{~mm}$ to $D=1.2 \mathrm{~mm}, R_{0}=4.0 \mathrm{~mm}$, frequency $f=0.5 \mathrm{MHz}$.

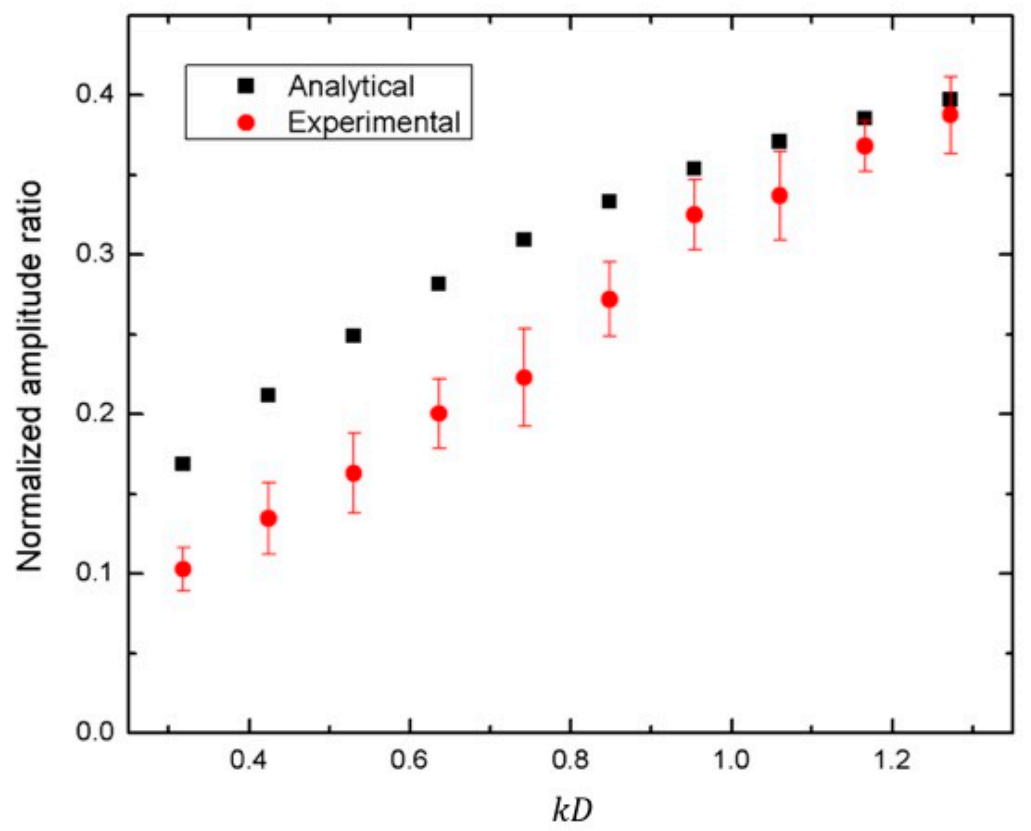

Figure 8. Normalized amplitude ratio between the scattered field and the incident field due to the interaction of the incident surface wave with a square cylindrical cavity: $D=0.3 \mathrm{~mm}$ to $D=$ $1.2 \mathrm{~mm}, R_{0}=4.0 \mathrm{~mm}$, frequency $f=0.5 \mathrm{MHz}$.

\section{Conclusions}

The application of reciprocity to the scattering of surface waves by a three-dimensional cavity of arbitrary shape in an elastic half-space has been shown in this article. We have introduced a new and elegant approach using the distributed loading to compute the far-field scattered waves generated by the interaction between the incident surface waves and the cavity. The explicit equations of the scattered fields produced by a spherical, a circular cylindrical (coin-shaped) and a square cylindrical cavity 
have been theoretically derived. In order to validate these theoretical predictions, the experimental work using the pulse-echo method was performed to measure the reflected echoes after the interaction of the incident waves with the cavities. We compared the normalized amplitude ratio, which is the ratio of the amplitude proportion of the scattered to the incident fields, obtained using experimental and theoretical studies. In terms of trends and magnitudes, the comparisons have been in excellent agreement for the case of a spherical cavity and good agreement for the cases of a circular and a cylindrical cavity. The limitations of the proposed analytical approximation regarding the degree of smoothness and the size of the cavity were also discussed.

Author Contributions: J.L. designed and performed the experiments. H.P. and Y.C. conceived the original idea. V.N. developed the theory and performed the computations with support from J.L. and H.P., D.K.D. verified the analytical methods. T.N. wrote the manuscript in consultation with Y.C. All authors discussed the results and contributed to the final manuscript.

Funding: This research has been supported by the Vietnam Foundation for Science and Technology Development (NAFOSTED) under grant reference 107.02-2019.21 and the National Research Foundation of Korea (NRF) grant funded by the Korea government (MIST) (no. NRF-2019R1G1A1004577, 2019R1A5A808320111).

Acknowledgments: Authors appreciate the support from Jan D. Achenbach at Northwestern University.

Conflicts of Interest: The authors declare no conflict of interest.

\section{References}

1. Hassan, W.; Veronesi, W. Finite element analysis of Rayleigh wave interaction with finite-size, surface-breaking cracks. Ultrasonics 2003, 41, 41-52. [CrossRef]

2. Cho, Y.; Rose, J.L. An elastodynamic hybrid boundary element study for elastic guided wave interactions with a surface breaking defect. Int. J. Solids Struct. 2000, 37, 4103-4124. [CrossRef]

3. Shi, F.; Lowe, M.J.S.; Skelton, E.A.; Craster, R.V. A time-domain finite element boundary integral approach for elastic wave scattering. Comput. Mech. 2018, 61, 471-483. [CrossRef]

4. Gilbert, F.; Knopoff, L. Seismic scattering from topographic irregularities. J. Geophys. Res. 1960, 65, 3437-3444. [CrossRef]

5. Mendelsohn, D.A.; Achenbach, J.D.; Keer, L.M. Scattering of elastic waves by a surface-breaking crack. Wave Motion 1980, 2, 277-292. [CrossRef]

6. Ogilvy, J.A. Wave scattering from rough surfaces. Rep. Prog. Phys. 1987, 50, 1553. [CrossRef]

7. Castaings, M.; Le Clezio, E.; Hosten, B. Modal decomposition method for modeling the interaction of Lamb waves with cracks. J. Acoust. Soc. Am. 2002, 112, 2567-2582. [CrossRef]

8. Wang, X.-M.; Ying, C.F. Scattering of Lamb waves by a circular cylinder. J. Acoust. Soc. Am. 2001, 110, 1752-1763. [CrossRef]

9. Arias, I.; Achenbach, J.D. Rayleigh wave correction for the BEM analysis of two-dimensional elastodynamic problems in a half-space. Int. J. Numer. Meth. Eng. 2004, 60, 2131-2146. [CrossRef]

10. Liu, W.; Cho, Y.; Phan, H.; Achenbach, J. Study on the scattering of 2-D Rayleigh waves by a cavity based on BEM simulation. J. Mech. Sci. Technol. 2011, 25, 797-802. [CrossRef]

11. Phan, H.; Cho, Y.; Achenbach, J.D. Application of the reciprocity theorem to scattering of surface waves by a cavity. Int. J. Solids Struct. 2013, 50, 4080-4088. [CrossRef]

12. Phan, H.; Cho, Y.; Achenbach, J.D. Validity of the reciprocity approach for determination of surface wave motion. Ultrasonics 2013, 53, 665-671. [CrossRef] [PubMed]

13. Phan, H.; Cho, Y.; Li, W. A theoretical approach to multiple scattering of surface waves by shallow cavities in a half-space. Ultrasonics 2018, 88, 16-25. [CrossRef] [PubMed]

14. Hesse, D.; Cawley, P. Defect detection in rails using ultrasonic surface waves. Insight-Non-Destr. Test. Cond. Monit. 2007, 49, 318-326. [CrossRef]

15. Tuan, H.-S.; Li, R.C.M. Rayleigh-wave reflection from groove and step discontinuities. J. Acoust. Soc. Am. 1974, 55, 1212-1217. [CrossRef]

16. Simons, D.A. Reflection of Rayleigh waves by strips, grooves, and periodic arrays of strips or grooves. J. Acoust. Soc. Am. 1978, 63, 1292-1301. [CrossRef] 
17. Kosachev, V.V.; Lokhov, Y.N.; Chukov, V.N. On the theory of scattering the rayleigh surface acoustic waves by a two-dimensional statistical roughness of a free solid surface. Solid State Commun. 1990, 73, 535-539. [CrossRef]

18. Gregory, R.D.; Austin, D.M. Scattering of waves by a semicylindrical groove in the surface of an elastic half-space. Q. J. Mech. Appl. Math. 1990, 43, 293-315. [CrossRef]

19. DeSanto, J.A. Exact boundary integral equations for scattering of scalar waves from perfectly reflecting infinite rough surfaces. Wave Motion 2008, 45, 918-926. [CrossRef]

20. Martin, P.A. Multiple Scattering: Interaction of Time-Harmonic Waves with N Obstacles; Cambridge University Press: Cambridge, UK, 2006.

21. Hao, S.; Strom, B.W.; Gordon, G.; Krishnaswamy, S.; Achenbach, J.D. Scattering of the Lowest Lamb Wave Modes by a Corrosion Pit. Res. Nondestruct. Eval. 2011, 22, 208-230. [CrossRef]

22. Mossessian, T.K.; Dravinski, M. Scattering of elastic waves by three-dimensional surface topographies. Wave Motion 1989, 11, 579-592. [CrossRef]

23. Moreau, L.; Caleap, M.; Velichko, A.; Wilcox, P.D. Scattering of guided waves by through-thickness cavities with irregular shapes. Wave Motion 2011, 48, 586-602. [CrossRef]

24. Lee, J.; Achenbach, J.D.; Cho, Y. Use of the reciprocity theorem for a closed form solution of scattering of the lowest axially symmetric torsional wave mode by a defect in a pipe. Ultrasonics 2018, 84, 45-52. [CrossRef] [PubMed]

25. Moreau, L.; Caleap, M.; Velichko, A.; Wilcox, P.D. Scattering of guided waves by flat-bottomed cavities with irregular shapes. Wave Motion 2012, 49, 375-387. [CrossRef]

26. Grahn, T. Lamb wave scattering from a circular partly through-thickness hole in a plate. Wave Motion 2003, 37, 63-80. [CrossRef]

27. Ewing, W.M.; Zardetzky, W.S.; Press, F. Elastic Waves in Layered Media; McGraw-Hill: New York, NY, USA, 1957; p. 55.

28. Achenbach, J.D. Wave Propagation in Elastic Solids; North-Holland Publishing Company: Amsterdam, The Netherlands, 1973.

29. Rayleigh, L. On Waves Propagated along the Plane Surface of an Elastic Solid. Proc. Lond. Math. Soc. 1885, s1-s17, 4-11. [CrossRef]

30. Phan, H.; Cho, Y.; Achenbach, J.D. Verification of surface wave solutions obtained by the reciprocity theorem. Ultrasonics 2014, 54, 1891-1894. [CrossRef]

31. Phan, H.; Cho, Y.; Le, Q.H.; Pham, C.V.; Nguyen, H.T.L.; Nguyen, P.T.; Bui, T.Q. A closed-form solution to propagation of guided waves in a layered half-space under a time-harmonic load: An application of elastodynamic reciprocity. Ultrasonics 2019, 96, 40-47. [CrossRef] 